\title{
Análisis de vulnerabilidad de las comunidades de Coris y Tablón, Provincia de Cartago, Costa Rica
}

\author{
Johan Córdoba Peraza
}

\section{Resumen:}

La investigación consiste en el análisis de vulnerabilidad en las comunidades de Coris de Cartago y Tablón de El Guarco ante el impacto de los desastres. Estas comunidades se sitúan dentro de la microcuenca del río Purires en la provincia de Cartago, Costa Rica. La microcuenca del río Purires, se ubica en el extremo oriental de la región central de Costa Rica; abarca 76, $2 \mathrm{~km}^{2}$ y forma parte de la sección alta de la cuenca del río Reventazón.

La metodología utilizada se enmarca dentro del paradigma de la Investigación Cualitativa de Acción Participativa desde el enfoque de la educación no formal. El objetivo general de la investigación, se centró en analizar las amenazas y la vulnerabilidad a las que están expuestas las comunidades de Coris y Tablón. Para ello fue necesario desarrollar un plan de acción participativo comunitario, que buscó implementar las recomendaciones para mejorar la capacidad de respuesta de las comunidades ante el impacto de los desastres.

Los resultados demostraron que en Coris se identificaron 18 amenazas, clasificadas en antrópicas, relacionadas con la contaminación ambiental, delincuencia y el vandalismo; socionaturales como deslizamientos y desbordamiento de ríos; y naturales, asociadas a sismos por fallamientos geológicos locales que afectan la microcuenca. En Tablón existen un total de 18 amenazas, clasificadas por su origen en socionaturales, de las cuales nueve corresponden a deslizamientos, seis a desbordamientos de ríos y una por erosión e inadecuadas prácticas de cultivo; una antrópica relacionada con el consumo de drogas y alcohol; y una natural asociada a sismos.

Los escenarios de riesgo seleccionados en Tablón y Coris se agruparon por su nivel de aceptabilidad en inadmisibles e inaceptables, aceptables y tolerables con el fin de disminuir su condición mediante recomendaciones desde la perspectiva de la gestión del riesgo para la prevención de los desastres. 
Palabras clave: Coris. Tablón. Vulnerabilidad. Amenaza. Escenarios.

\section{Abstract:}

The present research consists of an analysis of vulnerability in the communities of Coris of Cartago and Tablon of El Guarco against the impact of disasters. These communities are contained within the Purires river micro-watershed, in the province of Cartago, Costa Rica. The micro-basin of river Purires is located on the east side of the central region of Costa Rica; it extends $76,2 \mathrm{~km}^{2}$ and is part of the upper section of the Reventazon river basin.

The methodology is based on the Participative-Action Qualitative Research framework, within the informal education approach. The main objective of this research is centered upon analyzing the threats and vulnerability to which the communities of Coris and Tablon are exposed. For this, it was necessary to develop an community action plan that attempted to implement recommendations to improve the response capacity of the communities to disaster impacts.

Results report that in Coris there were 18 threats identified, classified as anthropogenic and related to environmental pollution, vandalism, crime. Furthermore, socionatural threats such as landslides and river flooding were identified, as well as natural threats, such as tectonic activity due to local geologic faults that affect the watershed. In Tablon a total of 18 threats were also identified, classified based on their origin as socionatural (of which nine correspond to landslides, five to river flooding and one to erosion and inadequate farming practices), anthropogenic (related to drug consumption and alcohol), and natural (tectonic activity).

The selected threat scenarios in Tablon and Coris were grouped according to their acceptance level into inadmissible and unacceptable, and acceptable and tolerable. This was done with the purpose of reducing its condition through recommendations from the perspective of risk management for disaster prevention.

Keywords: Coris. Tablón. Vulnerability. Hazard. Scenarios

Johan Córdoba Peraza (johancordoba78@gmail.com). Geógrafo, M.Sc. en gestión del riesgo en desastres. 


\section{INTRODUCCIÓN}

El presente artículo describe en forma resumida la metodología utilizada y los resultados obtenidos en el Trabajo Final de Investigación Aplicada (TFIA): Análisis de vulnerabilidad de las comunidades de Coris y Tablón en la microcuenca del río Purires, provincia de Cartago, Costa Rica. Esta investigación, se desarrolló durante el segundo semestre del año 2011 y el primer trimestre del año 2012, y fue presentada a la Comisión del Programa de Estudios de Posgrado en Gestión del Riesgo en Desastres de la Escuela Centroamericana de Geología de la Universidad de Costa Rica, en el mes de septiembre del 2012.

EI TFIA, fomó parte de un proyecto de investigación mucho más amplio sobre saneamiento ambiental; que fue propuesto y liderado por el Departamento de Agua y Saneamiento de Países en Desarrollo (SANDEC) del Instituto Federal Suizo en Ciencias Acuáticas y Tecnologías (EAWAG).

Asimismo, dicho TFIA se realizó gracias al apoyo académico, logístico y monetario de cinco instituciones: el Centro Nacional Suizo de Competencia en Investigación Norte-Sur (NCCR), la Swiss National Science Foundation (SNSF), la Agencia Suiza para el Desarrollo y Cooperación (SDC), la Facultad Latinoamericana de Ciencias Sociales con sede académica en Costa Rica (FLACSO-CR) y la Universidad de Costa Rica a través del Programa de Gestión Ambiental Integral (ProGAl-UCR).

El objetivo general del trabajo de investigación consistió en analizar la vulnerabilidad de las comunidades de Coris de Cartago y Tablón de El Guarco.

Asimismo, los objetivos específicos planteados fueron los siguientes:

- Caracterizar la microcuenca del río Purires desde un punto de vista socioambiental.

- Describir las amenazas naturales y socionaturales presentes en el área de estudio.

- Estimar la vulnerabilidad en la infraestructura comunal de Coris de Cartago y Tablón de El Guarco. 
- Proponer recomendaciones que se ajusten a las condiciones socioambientales de las comunidades de Coris y Tablón en la microcuenca del río Purires.

Todos los objetivos anteriormente citados, se cumplieron gracias a la participación activa de los miembros de las comunidades, las Asociaciones de Desarrollo Comunal y las ASADAs'.

Esta participación facilitó recopilar y clasificar la información sobre las áreas geográficas y los recursos de infraestructura comunal que, según sus pobladores, son susceptibles de manifestación e impacto de eventos peligrosos de origen natural, antrópico y socionatural. Esto, con el propósito de implementar medidas correctivas para disminuir las condiciones de vulnerabilidad existenes desde la perspetiva de la gestión del riesgo para la prevención de los desastres.

\section{METODOLOGÍA}

Para Kholer et al. (2004), existen varios enfoques para elaborar un análisis de vulnerabilidad; por esa razón, no se han establecido procedimientos estandarizados, y no hay un consenso definido en cuanto a los indicadores pertinentes por utilizar. Dado lo anterior, la selección de una determinada metodología para el análisis de vulnerabilidad, estará íntimamente relacionada con la escala de análisis y las características de los elementos bajo estudio (Melone, 2003).

El proceso metodológico utilizado en la investigación posee cuatro etapas de bajo costo económico, abiertas, dinámicas y enmarcadas dentro del paradigma de la Investigación Cualitativa de Acción Participativa desde el enfoque de la educación no formal ${ }^{2}$ las cuales se detallan de forma resumida a continuación:

La Etapa I consistió en la evaluación de las condiciones y las variables socioambientales más relevantes de la microcuenca del río Purires tales como: rasgos socioeconómicos y demográficos de la población que habita en las comunidades de

\footnotetext{
${ }^{1}$ Asociaciones Administradoras de Sistemas de Agua Potable y Saneamiento.

${ }^{2}$ Según Trilla (1992), es un proceso educativo voluntario, pero intencionado, planificado, pero permanentemente flexible, que se caracteriza por la diversidad de métodos, ámbitos y contenidos en los que se aplica. Para lograr lo anterior, fue necesario trabajar en conjunto con una profesional en dicho campo.
} 
estudio; rasgos morfométricos de la microcuenca, clima y uso de la tierra, así como el tipo de suelo; geomorfología y geología presente en el área de estudio.

La Etapa II se basó en el trabajo de campo, mediante recorridos y giras planificadas a las comunidades de Coris y Tablón, así como al resto del área de la microcuenca del río Purires, con el objetivo de recolectar información no documentada en fuentes bibliográficas y cartográficas existentes.

En la Etapa III se aplicó el instrumento metodológico diseñado por Toro (2002) denominado: Metodología para el análisis de riesgo y vulnerabilidad en sistemas de agua potable y saneamiento, cuyo propósito final es obtener resultados en valores porcentuales (resultados cuantitativos) a partir de información cualitativa (Céspedes, 2011).

Según la FICR (2006), el análisis de vulnerabilidad consiste en recopilar, analizar y sistematizar, de una forma estructurada y lógica, información sobre la vulnerabilidad de una comunidad a una determinada amenaza. La información recopilada, sirve entonces, para diagnosticar los riesgos principales y las capacidades actuales de la comunidad y lleva, en último término, a la preparación de actividades dirigidas a reducir la vulnerabilidad de la población ante posibles desastres y así, incrementar su capacidad de supervivencia y recuperación.

Para lograr lo anterior, fue necesario el desarrollo de talleres de trabajo, donde la participación de los hombres y mujeres, tanto adultos como jóvenes, que forman parte de las comunidades e integran organizaciones de diverso índole en ambos pueblos, fue clave y de suma importancia. La aplicación de la herramienta metodológica seleccionada proporcionó criterios de intervención, mediante la construcción de escenarios de riesgo que facilitaron elegir, identificar y valorar (cualitativamente y cuantitativamente) las amenazas, el riesgo y la vulnerabilidad que presentaron no sólo los sistemas de abastecimiento y saneamiento de agua, sino toda la infraestructura comunal (escuelas y viviendas, puentes, templos y salones comunales, colegios, caminos y líneas de transmisión eléctrica), cuya inclusión en el análisis fue considerada como importante por los participantes de los talleres de trabajo.

Por último, la etapa IV comprendió la organización, síntesis y presentación final de los resultados obtenidos en las etapas I, II y III, a partir de lo cual se defi- 
nieron estrategias y recomendaciones desde la perspectiva de la gestión del riesgo para la prevención de los desastres, en busca de mejorar la calidad de vida de las personas que habitan en las comunidades de estudio.

\section{ÁREA DE ESTUDIO}

La microcuenca del río Purires se localiza en el extremo oriental de la región central de Costa Rica. Limita al norte con los cerros de la Carpintera; al oeste y al sur, con las estribaciones de la cordillera de Talamanca y al este, con las ciudades de Cartago y Tejar de El Guarco (Figura 1). La microcuenca cubre un área de $76,2 \mathrm{~km}^{2}$ y se emplaza en la sección alta de un sistema hidrográfico de mayores dimensiones (2 $950 \mathrm{~km}^{2}$ ): la cuenca del río Reventazón-Parismina que desemboca en el Mar Caribe. Esta cuenca es una de las más importantes de Costa Rica, pues allí se genera el $25 \%$ de la energía hidroeléctrica del país, abastece de agua potable al $50 \%$ de la población del Área Metropolitana de San José y se produce el $11 \%$ del valor total de las exportaciones locales (Sogreah Ingenierie et al., 2000).

Figura 1: Ubicación del área de estudio
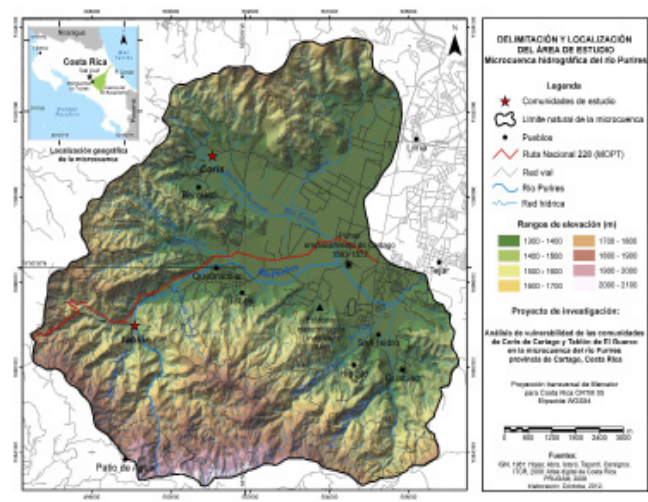

Fuente: Elaboración propia, 2013.

Asimismo, a pesar de que la zona general de estudio fue la microcuenca del río Purires, se consideraron también las comunidades de Coris de Cartago y Tablón de El Guarco, en las cuales se elaboró el análisis de vulnerabilidad; por tanto, es necesario establecer su ubicación geográfica como ejes de referencia espacial.

El pueblo de Coris de Cartago (Figura 1) se localiza sobre los $1400 \mathrm{~m}$ de altitud y se ubica al noroeste de la microcuenca, aproximadamente a $3 \mathrm{~km}$ del pueblo 
de Quebradilla. Administrativamente pertenece al distrito de Quebradilla del cantón central de Cartago en la provincia de Cartago.

Por su parte, el pueblo de Tablón de El Guarco se sitúa sobre los $1600 \mathrm{~m}$ de altitud y se ubica en el extremo suroeste de la microcuenca, aproximadamente a 2, $5 \mathrm{~km}$ de distancia del pueblo de Tobosi. En términos político - administrativos pertenece al distrito de Tobosi del cantón de El Guarco, provincia de Cartago.

Dado su contexto geográfico y social, ecológico y climático, así como geológico y geomorfológico, la microcuenca del río Purires está expuesta a un escenario de múltiples amenazas compuestas por la combinación de eventos naturales como sismos, actividad volcánica y eventos hidrometeorológicos; amenazas socionaturales les como deslizamientos, erosión y desbordamientos de la red hídrica y amenazas antrópicas como derrames de sustancias peligrosas y contaminación del agua, aire y suelo, generada por la actividad industrial, comercial, agropecuaria y residencial ubicada tanto dentro como fuera de la microcuenca.

En términos morfométricos la microcuenca del río Purires presenta un factor de forma de 0, 76 (forma casi circular) y un patrón de drenaje con forma dendrítica de densidad regular (Zúñiga, 1993) que cubre un área de 76,2 km², por lo cual se puede clasificar como una microcuenca pequeña.

El clima del área de estudio es particular, ya que es afectado por la influencia directa e indirecta de eventos hidrometeorológicos tanto, en el Mar Caribe como en el Oceáno Pacífico. Según Solano y Villalobos (2001), este clima se clasifica como Valle Central 2 (VC2), ya que registra precipitaciones moderadas cercanas a los 2000 mm por año (con 128 días de lluvia y sólo un mes seco durante el año), así como temperaturas frescas con máximas promedio de $26^{\circ} \mathrm{C}$ y mínimas promedio de $15^{\circ} \mathrm{C}$.

En cuanto a la geología, Carrillo \& Carrillo (2005), indican que la microcuenca está formada por litologías de origen sedimentario (Formación Coris y San Miguel) y volcánico (Grupo Aguacate) muy meteorizadas, con espesores que oscilan entre los 15 m y 500 m (Berrangué \& Whittaker, 1977; Denyer \& Arias, 1991) y que fueron depositadas alternadamente desde la época del Terciario. Estas litologías se ubican en los sectores norte, oeste y sur de la microcuenca (Figura 2). 
Figura 2: Geología del área de estudio

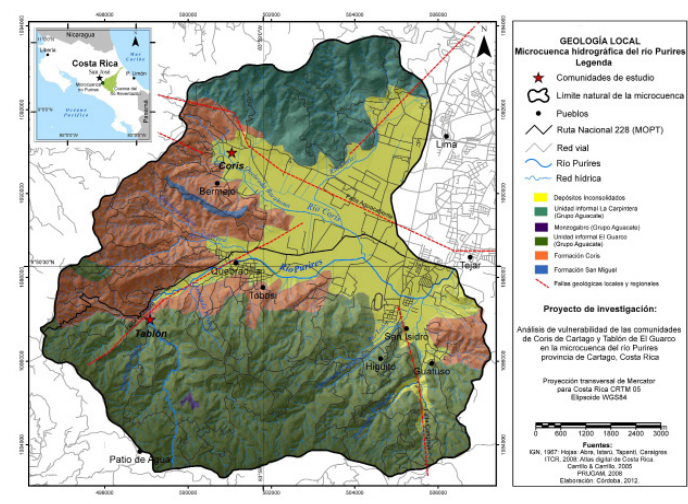

Fuente: Elaboración propia, 2013.

Además de las litologías anteriormente mencionadas, existen depósitos inconsolidados recientes del período Cuaternario como coluvios y aluviones muy alterados por la influencia del intemperismo, éstos se ubican en el sector central de la microcuenca donde la topografía es relativamente plana (Figura 2). Según Carrillo \& Carillo (2005), dichos materiales se caracterizan porque no superan los 5 $\mathrm{m}$ de espesor y por presentar matrices de plasticidad media y alta.

La geomorfología del área de estudio debe su desarrollo a la combinación de eventos geológicos (sismos y actividad volcánica); procesos denudacionales y fluviales intensos, que están generando unidades del relieve compuestas por áreas con pendientes fuertes y escarpes propensos a deslizarse (Carrillo \& Carrillo, 2005); así como zonas planas y onduladas en las que la red hídrica divaga y se desborda constantemente en período lluvioso, lo cual representa una potencial amenaza socionatural para las poblaciones, los cultivos y la infraestructura instalada en las partes bajas de la microcuenca.

El relieve de la zona de estudio está compuesto en su mayoría ( $56 \%$ del total del área de estudio) por pendientes planas y medias entre los $0^{\circ}$ y los $30^{\circ}$ de inclinación. Sin embargo, el restante $44 \%$ está conformado por un relieve inestable de pendientes fuertes y muy fuertes (mayores a los $30^{\circ}$ de inclinación), localizadas geográficamente al sur de la microcuenca (Figura 2). Dicho relieve, se compone de 
litologías volcánicas del período Terciario, muy meteorizadas y propensas a deslizarse cuando se combinan con eventos detonantes y disparadores como lluvias intensas y eventos sísmicos de magnitudes considerables (Carrillo \& Carrillo, 2005).

La situación anterior, evidencia una amenaza potencial para las personas, los cultivos y la infraestructura ubicada, particularmente, en los sectores que presentan una topografía con pendientes fuertes, pues los temblores, en áreas sismicamente activas (similar al área de estudio), ponen en movimiento las laderas inestables en forma de deslizamientos (Peraldo \& Acevedo, 2010).

El tipo de suelo predominante (un 92\% del total del área de la microcuenca) es inceptisol (suelo incipiente) con texturas limosas, arenosas y arcillosas (Carrillo \& Carrillo, 2005), producto de la meteorización de rocas sedimentarias y volcánicas de los períodos Terciario y Cuaternario.

Finalmente, el paisaje del área está tapizado por un mosaico de coberturas vegetales y un uso de la tierra variado pero con una marcada tendencia a la actividad agropecuaria. Según el PRUGAM (2010), los usos de la tierra predominantes en la zona de estudio son: bosques secundarios $(41,27 \%)$, pastos y áreas deforestadas $(25,19 \%)$, así como uso mixto $(16,43 \%)$ e invernaderos para la producción de hortalizas y plantas ornamentales $(6,70 \%)$.

\section{RESULTADOS}

El tema y las acciones relacionadas con los desastres y la gestión del riesgo, es un campo que cuenta con larga trayectoria de investigación, bibliografía, enfoques teóricos y experiencias en la gestión (Fernández, 1996). Por tal razón, en este apartado se define brevemente el concepto asociado a la vulnerabilidad, dado a que éste, es utilizado en reiteradas ocasiones a lo largo del presente documento.

La vulnerabilidad de las sociedades ante el impacto de los desastres, se produce por la interacción de un conjunto de factores como la falta de planificación y la ausencia de políticas a largo plazo para el ordenamiento del territorio, la debilidad institucional y la intensificación del uso de la tierra, así como la explotación descontrolada de los recursos naturales, el incremento acelerado de la población y la presencia de condiciones socioeconómicas desfavorables, sobre todo, en comunidades empobrecidas (Buch \& Turcios, 2003). 
Además, la Ley Nacional de Emergencias y Prevención del Riesgo de Costa Rica (La Gaceta No 8, 11 de enero de 2006: Ley N8488) plantea que la vulnerabilidad es una condición intrínseca de ser impactado por un suceso a causa de un conjunto de condiciones y procesos físicos, sociales, económicos y ambientales. Se determina por el grado de exposición y fragilidad de los elementos susceptibles de ser afectados, la población, sus haberes, las actividades de bienes y servicios, el ambiente y la limitación de su capacidad para recuperarse.

Una vez definido el concepto de vulnerabilidad, se procederá a presentar los resultados obtenidos del análisis de vulnerabilidad realizado en las comunidades de Coris y Tablón, el cual inició con el inventario comunal de recursos para saber qué recursos e infraestructuras existían en cada comunidad.

El objetivo del inventario fue estimular la discusión sobre el estado, la calidad, la antigüedad, el tipo de materiales y la ubicación geográfica de los recursos para identificar las amenazas o peligros en un futuro cercano (Toro, 2002; Córdoba, 2012). Los inventarios se elaboraron y plasmaron en catálogos y mapas de percepción, que fueron realizados por los participantes de los talleres de trabajo.

Las comunidades de Coris de Cartago (Figura 3) y Tablón de El Guarco (Figura 4) disponen de recursos infraestructurales (viviendas, caminos, iglesias, escuelas y salones comunales), así como de organizaciones comunales (mujeres, desarrollo integral, deportes, educativas y ASADAs) los cuales generaron condiciones para facilitar el diálogo con sus respectivos representantes y líderes, en términos de dirigir esfuerzos y recursos hacia la identificación de factores de riesgo a nivel local.

Lo anterior permitió definir acciones para disminuir el riesgo desde la construcción local con perspectiva en el mediano y largo plazo, y que así los actores sociales puedan participar activamente en la gestión adecuada de sus recursos comunales, humanos y ambientales (Brenes \& González, 1996); ya que según Santos (1986), la casa, el lugar, el trabajo, los puntos de encuentro, los caminos que unen entre sí estos puntos, son elementos pasivos (con significancia) que condicionan la actividad del serhumano y comandan su práctica social.

En Coris de Cartago, la infraestructura gestionada y construida por la propia comunidad representa el $70 \%$ del total del inventario realizado a través de los talleres de trabajo. Estos están compuestos por el centro educativo y el salón de cate- 
quesis, el templo católico y el salón comunal, la plaza de deportes, los vestidores y el acueducto. Además el inventario también incluyó la infraestructura vial (caminos principales, vecinales y puentes), bajo la administración de la Municipalidad de Cartago y los órganos adscritos al MOPT como el CONAVI o el COLOSEVI?3.

Dicha infraestructura beneficia tanto a los pobladores de Coris como a personas de otras comunidades (Tablón, Quebradilla, Bermejo y Tobosi), pues facilitan la comunicación y el desplazamiento diario de las personas a los centros de salud, educación, comercio, zonas de cultivo y lugares de trabajo, situados tanto dentro como fuera de los límites de la microcuenca. Estos recursos representan el $30 \%$ del total del inventario de la infraestructura de la comunidad.

Por su parte, el inventario de recursos en el pueblo de Tablón de El Guarco demostró que la infraestructura comunal gestionada y construida por la propia comunidad representa el 60\%. Esta está compuesta por el templo católico, el salón de catequesis, la escuela y el salón comunal, el campo de fútbol, la oficina de la ASADA y los tanques de almacenamiento de agua.

El restante $40 \%$ de los recursos se ha construido mediante la intervención estatal, comunal y municipal, la cual está compuesta por la comandancia policial, la Ruta Nacional 228, cinco puentes, así como una red de caminos vecinales y privados que facilitan la comunicación y el transporte de las personas a pueblos, fincas, y centros educativos y de salud cercanos a la comunidad de Tablón durante la mayor parte del año, pues en período de lluvias, estas rutas son afectadas por deslizamientos y hundimientos que las inhabilita parcialmente.

Brenes y González (1996) plantean que para generar organización y sentido de comunidad, no basta con compartir un espacio geográfico común, ya que se necesita establecer vínculos por medio de un sentido de pertenencia e identidad con el entorno físico y social. Asimismo, el hecho de que quienes viven en un mismo lugar compartan una preocupación común y trabajen colectivamente para resolverlas, es lo que permite articularse como comunidad, situación que se prensenta tanto en Coris de Cartago como en Tablón de El Guarco. Por tal razón, se recopiló

\footnotetext{
${ }^{3}$ Los Consejos Locales de Seguridad Vial (Administrados conjuntamente por las comunidades y las municipalidades y asesorados por las Municipalidades) son órganos adscritos al Ministerio de Obras Públicas y Transportes de Costa Rica (MOPT) y el Consejo Nacional de Vialidad.
} 
información sobre las organizaciones comunales existentes en ambos lugares.

En Coris las organizaciones se gestaron entre 1980 y 1990 para satisfacer necesidades de los pobladores relacionadas con el acceso a los servicios y la construcción de infraestructura comunal básica. Estas organizaciones son de índole religiosa, educativa, deportiva y de desarrollo comunal, así como de gestión del recurso hídrico para el consumo humano.

Sin embargo, no se tuvo referencia e información sobre alguna organización relacionada con el tema de la gestión del riesgo, la prevención y la respuesta ante emergencias de origen natural, socionatural y antrópicas.

Es importante destacar esta situación, ya que podría influir en un proceso de construcción histórica de la vulnerabilidad de la comunidad de Coris ante el impacto de eventos o emergencias futuras, debido a la falta de experiencia, protocolos, planes y estrategias de prevención y de respuesta ante la manifestación de eventos amenazantes por parte de las personas que habitan el pueblo de Coris.

Por su parte, las organizaciones comunales en Tablón se gestaron en las décadas del 1970 hasta 1990, con el propósito de satisfacer las necesidades de los pobladores del lugar relacionadas con el acceso a los servicios básicos y la construcción de infraestructura comunal básica. Dichas organizaciones comunales, también se dedican a actividades religiosas, educativas, deportivas, agrícolas y de desarrollo comunal, así como a la administración y protección del recurso hídrico para consumo humano, que beneficia tanto a los pobladores de la comunidad de Tablón, como a las personas de comunidades cercanas como Tobosi de El Guarco y Quebradilla de Cartago.

Es importante mencionar que en el mes de agosto del año 2011, se formó un pequeño comité comunal de emergencias adscrito a la Asociación de Desarrollo Integral de Tablón, con el propósito de integrarse en el futuro al comité distrital de emergencias de Tobosi, tal y como lo sugiere la Ley Nacional de Emergencias y Prevención del Riesgo de Costa Rica (La Gaceta № 8, 11 de enero de 2006: Ley N ${ }^{\circ}$ 8488).

La información referente a las organizaciones identificadas en las comunidades de Tablón y Coris, proporcionó un panorama para establecer relaciones de trabajo desde el ámbito de la gestión del riesgo para la prevención de los desastres 
(Escalante et al., 2006). Dicha sitación, favoreció las condiciones para la creación de futuros "sistemas de prevención y atención de desastres", de modo que toda la responsabilidad asociada a tal trabajo, no recaiga en un solo organismo centralizado, sino que se delegue a diversos órganos (Escalante et al., 2006) como instituciones públicas y privadas, no gubernamentales, académicas y grupos comunales.

Se ha mencionado en párrafos anteriores que la microcuenca del río Purires y por tanto las comunidades de Coris de Cartago y Tablón de El Guarco están situadas en un área con presencia de múltiples amenazas naturales (fallamientos geológicos locales y regionales activos, eventos hidrometeorológicos y peligros volcánicos) y socionaturales (deslizamientos, flujos de lodo, inundaciones y desbordamientos de la red hídrica) que históricamente las han impactado, generando pérdidas humanas y económicas en la infraestructura, cultivos y ganadería.

Así, se realizó un inventario de amenazas o eventos peligrosos que han afectado históricamente a ambas comunidades. El inventario se elaboró con base en la experiencia de las personas que participaron en los talleres de trabajo, pues son ellos los que poseen la memoria histórica del pueblo, y por supuesto, de los eventos naturales y socionaturales que con más frecuencia y capacidad de daño se han presentado en el pasado (Toro, 2002).

En cada una de las comunidades de estudio (Figuras 3 y 4 ) se identificaron 18 amenazas ${ }^{4}$ clasificadas por su nivel de significancia (dados sus valores a la frecuencia, área de impacto y magnitud) en: altas (valores entre 6-9, color rojo), medias (valores entres 3-5, color amarillo) y bajas (valores entre 1-3, color verde); así como por su origen en: socionaturales, naturales y antrópicas (Cuadro 1).

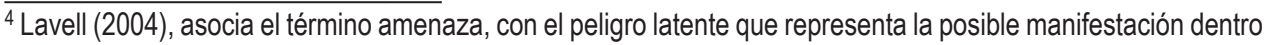
de un período de tiempo y en un territorio particular, de un evento de origen natural, socio-natural o antropogénico, que puede producir efectos adversos en las personas, la producción, la infraestructura, los bienes, los servicios y el ambiente.
} 
Cuadro 1: Clasificación de las amenazas por su origen en las comunidades de Coris y Tablón

\begin{tabular}{|c|c|c|c|c|}
\hline Tipos de amenazas & \multicolumn{2}{|c|}{ Coris } & \multicolumn{2}{c|}{ Tablón } \\
\hline Socionaturales & 7 & $39 \%$ & 16 & $90 \%$ \\
\hline Naturales & 1 & $5 \%$ & 1 & $5 \%$ \\
\hline Antrópicas & 10 & $56 \%$ & 1 & $5 \%$ \\
\hline Totales & $\mathbf{1 8}$ & $\mathbf{1 0 0} \%$ & $\mathbf{1 8}$ & $\mathbf{1 0 0 \%}$ \\
\hline
\end{tabular}

Fuente: Elaboración propia, 2013.

Según el Cuadro 1 las amenazas socionaturales en la comunidad de Tablón representan el $90 \%$ y en Coris, el 39\%. Estas amenazas están relacionadas principalmente con deslizamientos en áreas montañosas con pendientes fuertes (norte y sur de la microcuenca), erosión por diversas prácticas agrícolas, deforestación y desbordamiento de la red hídrica que atraviesa ambos pueblos. Dichas amenazas, se originan por la interacción de las variables ambientales de la zona de estudio con procesos históricos de intervención antrópica, ya que según Zusman (2002), las formas pasadas condicionan las acciones, las representaciones y la producción de formas presentes y futuras.

Figura 3: Amenazas y escenarios de riesgo identificados en la comunidad de Coris de Cartago
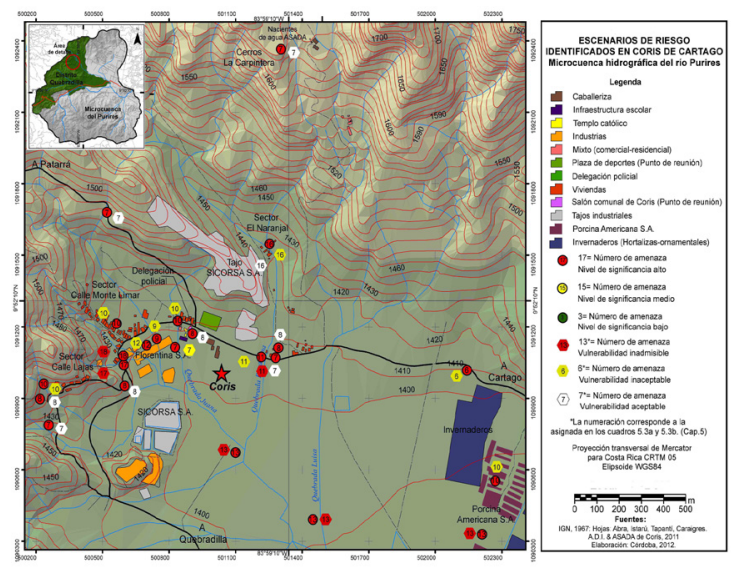

Fuente: Elaboración propia, 2013. 
Se mencionó en el párrafo anterior que las zonas cercanas a los centros de población de Coris y Tablón presentan altos grados de suceptibilidad a desbordamientos de los ríos y las quebradas, flujos de lodo y deslizamientos que se activan en períodos prolongados de lluvias, así como en eventos sísmicos con magnitudes considerables, lo cual ha provocado daños y pérdidas materiales en caminos y puentes, áreas de cultivos, acueductos y líneas de transmisión eléctrica.

Algunos de los factores que han influido en dicha suceptibilidad son el tipo de clima del área de estudio (el cual presenta precipitaciones cercanas a los 2000 $\mathrm{mm}$ por año), una topografía de pendientes fuertes (mayores a los $35^{\circ}$ de inclinación) con litologías muy meteorizadas, así como la presencia de suelos blandos y arcillosos con alta plasticidad. Lo anterior, aunado a grandes extensiones de terreno, cuyas coberturas vegetales han sido eliminadas como resultado del impacto de prácticas sociales y agrícolas íntensas que se derivan de la búsqueda de ganancia en el sentido económico (Lavell, 1996) y que privan la conservación del suelo, como un elemento básico para la productividad agrícola, así como un recurso escaso que se tiene que proteger y conservar para las generaciones futuras (Álvarez et al., 2010).

Figura 4: Amenazas y escenarios de riesgo identificados en la comunidad de Tablón de El Guarco
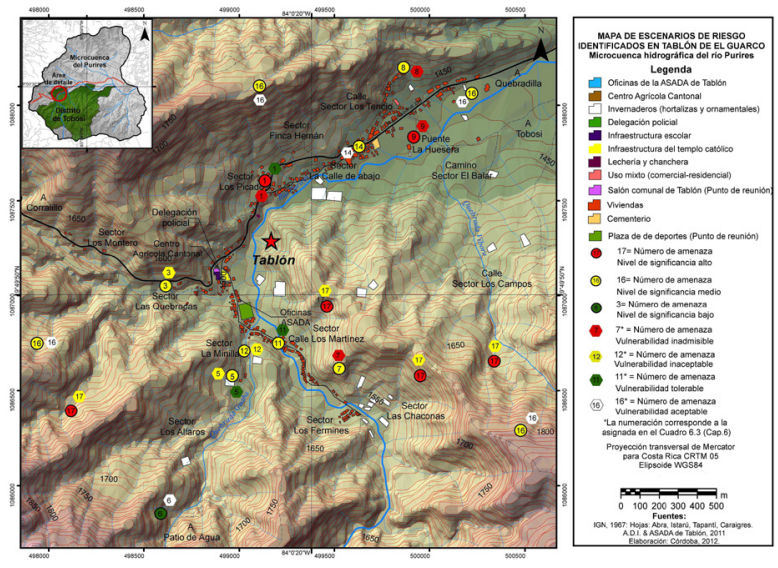

Fuente: Elaboración propia, 2013. 
Según el cuadro 1 y la Figura 5 es posible apreciar una marcada diferencia entre la cantidad de amenazas antrópicas identificadas en el pueblo Coris y en la comunidad de Tablón de El Guarco. En Coris, las amenazas antrópicas representan el $56 \%$ del total; es decir, 10 amenazas están asociadas a la contaminación del suelo (botaderos clandestinos), del aire (malos olores y polvo derivado de las fábricas de cerámica) y del agua (descargas residuales industriales a las quebradas y ríos). Se presentan, además, accidentes en la manipulación de sustancias tóxicas por actividades industriales y productivas, así como puentes en mal estado, delincuencia e inseguridad por la falta de vigilancia policial.

En tanto, en el pueblo de Tablón de El Guarco se identificó sólo una amenaza antrópica que representa el $5 \%$ del total. Esta amenaza está asociada a la inseguridad y al consumo de drogas ilegales (Figura 5).

Por consiguiente, se infiere que el registro en el número de amenazas antrópicas (especialmente aquellas relacionadas con sustancias peligrosas y contaminación), descritas por los participantes de los talleres en Coris, se podría deber a la cercanía geográfica de la comunidad de Coris respecto a la zona industrial de Cartago, donde se encuentran instalados multiples establecimientos industriales, clasificados por el Ministerio de Salud de Costa Rica como Tipo A 5 (riesgo alto).

Entre ellos destacan la extracción de cal, la producción de insumos agrícolas, abonos, papel, alimentos para mascotas, granjas avícolas y porcinas, aserraderos, fábricas de pinturas, industrias farmacéuticas y productoras de fibra de vidrio (Ministerio de Salud, Área Rectora de El Guarco, com. esc., 2011).

\footnotetext{
5 Establecimientos 0 actividades de riesgo alto: aquellas actividades o establecimientos que por sus características representan o pueden representar un riesgo potencial en forma permanente a la salud de las personas 0 al ambiente (La Gaceta № 42 del 29 de febrero del 2000: Decreto Ejecutivo № 34728-S.).
} 
Figura 5: Clasificación de las amenazas por su origen en las comunidades de Coris y Tablón

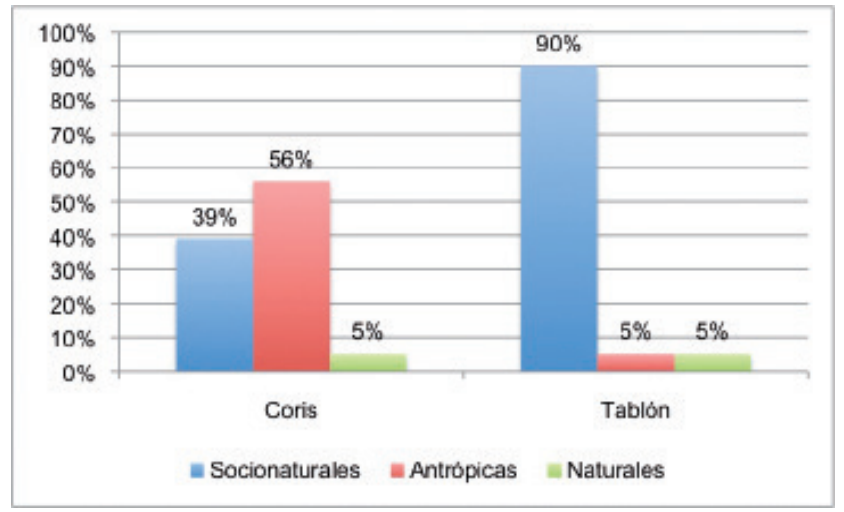

Fuente: Elaboración propia, 2013.

Por su parte, en el pueblo de Tablón la presencia y reporte de eventos y emergencias por contaminación de residuos industriales líquidos y sólidos es nula, dado que esta comunidad se ubica aproximadamente a $10 \mathrm{Km}$ de distancia de la zona industrial anteriormente mencionada.

En cuanto a las amenazas naturales, tal y como se muestra en el Cuadro 1 y la Figura 5, en ambas comunidades se identificó sólo una amenaza natural (5\%) asociada a sismos por fallamientos locales y regionales activos (Figura 2).

Es pertinente mencionar que dichas fallas geológicas han generado sismos destructivos como el ocurrido el 2 de septiembre de 1841 y 4 mayo de 1910 , los cuales destruyeron gran parte de las edificaciones de la ciudad de Cartago y generó gran cantidad de muertes y daños considerables en la infraestructura, tanto de Cartago, como de San José, Desamparados, Curridabat, Tres Ríos y Paraíso (Barquero, 2010; Peraldo \& Acebedo, 2010).

Estos sismos se caracterizaron por presentar sus hipocentros a poca profundidad de la superficie terrestre con magnitudes oscilaron entre 4 y 6 grados en la escala Richter; esto, aunado al relieve de pendientes fuertes (propenso a deslizarse al igual que la topografía del área de estudio) y un tipo de suelo blando y poco consolidado (que amplifica las ondas sísmicas), representa una importante amenaza, 
tanto para las personas como para la infraestructura de los pueblos situados dentro de la microcuenca del río Purires.

Por otra parte, la evaluación de las amenazas en las comunidades de Coris y Tablón se realizó utilizando dos períodos de tiempo diferentes, que fueron propuestos y elegidos por las personas que participaron en los talleres de trabajo en ambas comunidades.

Los participantes a los talleres de trabajo en la comunidad de Coris realizaron el inventario y la evaluación de las amenazas según un período de tiempo que abarcó un año (enero 2010 - diciembre 2010). A cada amenaza seleccionada se le realizó un análisis específico con base en tres variables de estudio: frecuencia, magnitud y área de impacto.

La elección de dicho período por parte de las personas se justificó aduciendo "que no recuerdan" que en años anteriores al 2010, la cotidianidad en la comunidad haya sido "severamente" afectada por la manifestación recurrente de eventos naturales o socionaturales. Sin embargo, se considera que lo anterior puede reflejar un desconocimiento o resistencia, por parte de las personas que participaron en los talleres de trabajo, a reconocer la recurrencia histórica de eventos que han afectado algunas zonas o la totalidad del área de estudio. La razón de estas condiciones puede estar asociada una corta memoria histórica, así como una percepción del riesgo desde una visión territorial y social muy acotada al espacio comunal (CEPAL, 2005) y desligada de la totalidad del área de la microcuenca del río Purires.

A diferencia de Coris, la evaluación de las amenazas en la comunidad de Tablón se realizó utilizando un período de tiempo que abarcó 15 años (desde el año de 1996 hasta el año 2011). A cada amenaza seleccionada también se le realizó un análisis específico utilizando las mismas variables que en el pueblo de Coris (frecuencia, magnitud y área de impacto).

La elección del período de tiempo se justificó aduciendo que desde el año de 1996 (año en el que Costa Rica fue impactada por el efecto indirecto del huracán César), se han presentado una serie de eventos recurrentes como desbordamientos y deslizamientos, que han afectado la infraestructura vial (caminos y puentes) y residencial del pueblo de Tablón y alrededores. 
Por otra parte, al sumar los valores asignados a la frecuencia, magnitud y área de impacto de cada amenaza seleccionada, se obtuvo el nivel significancia. Este evidenció que en la comunidad de Coris (Figura 6) el $61 \%$ de los eventos peligrosos se clasificó con un alto nivel de significancia; el 33\% se clasificó con un nivel medio y el restante $6 \%$, de baja significancia, dada la combinación de valores asignados a las tres variables de estudio en un período de un año (Figura 6).

En tanto, en la comunidad de Tablón de El Guarco las amenazas se clasificaron en un $67 \%$ con una significancia media; en un $22 \%$, con una alta significancia y el restante $11 \%$, de baja significancia dada su frecuencia, magnitud y área de impacto en un período análisis que abarcó 15 años (Figura 6).

Figura 6: Clasificación de las amenazas en las comunidades de Coris y Tablón dado su nivel de significancia

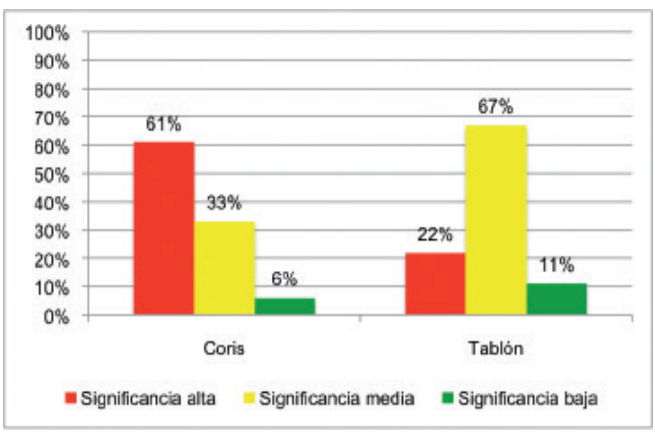

Fuente: Elaboración propia, 2013.

Sin embargo, para complementar la clasificación anterior se elaboró otra clasificación agrupando las amenazas por comunidad, nivel de significancia y por su origen; es decir, en socionaturales, antrópicas y naturales. Dado lo anterior, del total de amenazas socionaturales seleccionadas en Coris, el $29 \%$ presentó un nivel de significancia alto; el $57 \%$ de significancia media y el restante $14 \%$ de significancia baja (Figura 7).

Además, de las diez amenazas antrópicas identificadas, el 90\% de éstas se clasificó con una alta significancia, debido a la combinación de altos valores designados a la frecuencia, magnitud y área de impacto. En tanto, el restante $10 \%$ se 
clasificó como amenazas con un nivel de significancia medio. Por último, el 100\% de las amenazas naturales asociadas a sismos se clasificó con un nivel de significancia medio.

Figura 7: Clasificación de las amenazas por su origen y nivel de significancia en la comunidad de Coris

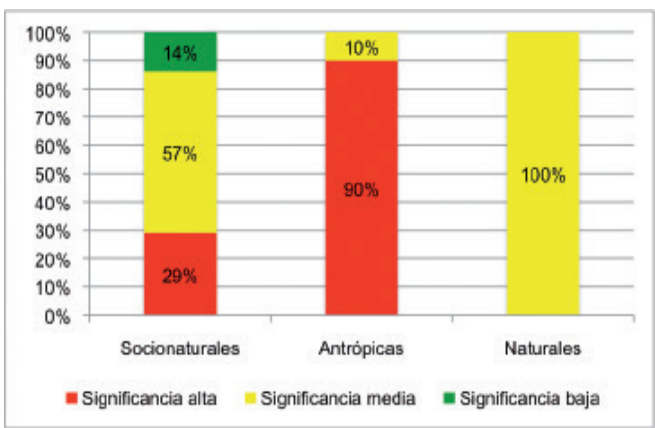

Fuente: Elaboración propia, 2013.

Según la figura 8, del total de eventos socionaturales peligrosos identificados en la comunidad de Tablón, el $25 \%$ presentó un nivel de significancia alto; el $63 \%$, un nivel de significancia medio y el $13 \%$, un nivel bajo de significancia. En tanto, las dos restantes amenazas (naturales y antrópicas) se clasificaron con niveles medios de significancia dado los valores asignados a la frecuencia, magnitud y área de impacto.

Figura 8: Clasificación de las amenazas por su origen y nivel de significancia en la comunidad de Tablón

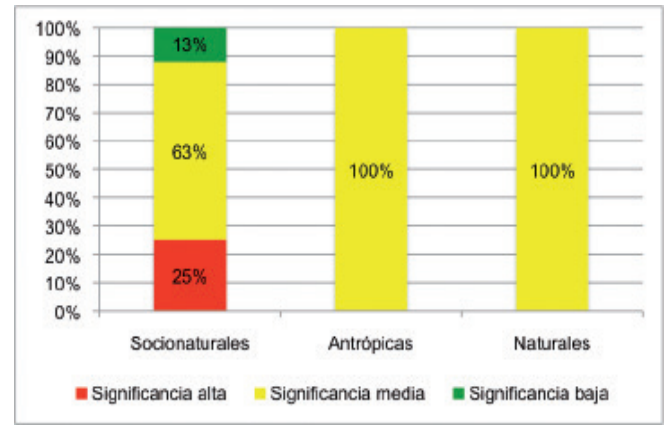

Fuente: Elaboración propia, 2013. 
Un vez definidas y categorizadas las amenazas en ambas comunidades (por su nivel de significancia), se procedió a establecer los escenarios de riesgo. Según Toro (2002) un escenario de riesgo es la representación de la interacción de las amenazas, el riesgo y las condiciones de vulnerabilidad en un territorio y en un período de tiempo dado.

Por lo tanto, este escenario debe permitir identificar los tipos de daños y las pérdidas, que pueden producirse en caso de presentarse un evento peligroso, bajo condiciones de vulnerabilidad específicas (Luengas, 2008).

Los escenarios de riesgo en cada comunidad de estudio se determinaron mediante la elaboración de una matriz construida a partir de las amenazas seleccionadas y el inventario de los recursos comunales anteriormente descritos. Estos escenarios se estimaron relacionando los recursos y las amenazas, y determinando las intersecciones (en una matriz) por medio de una $(\mathrm{x})$.

Además, con el fin de clasificar la gravedad relativa del riesgo que presentó cada escenario seleccionado y así definir la necesidad de intervenirlo y estimar la cantidad y el tipo de recursos humanos y materiales para ello, los escenarios de riesgo se clasificaron por su nivel de aceptabilidad (Figuras 9 y 10).

Figura 9: Clasificación de aceptabilidad escenarios de riesgo Coris

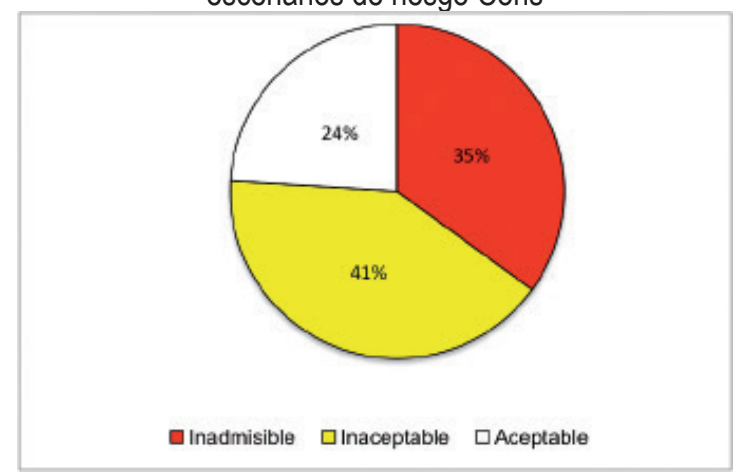

Fuente: Elaboración propia, 2013.

En la comunidad de Coris de Cartago se definieron un total de 17 escenarios de riesgo (Figura 9), compuestos todos ellos por amenazas antrópicas. Estos 
por su nivel de aceptabilidad se categorizaron de la siguiente manera: un $35 \%$ de los escenarios presentaron un nivel inadmisible, un $41 \%$ fueron clasificados como inaceptables y el restante $24 \%$ de los escenarios, como aceptables (Figura 9).

Dada la descripción anterior, se establece que en primer orden de intervención para disminuir su condición mediante el uso de recuros y sugerencias técnicas se incluyen 6 escenarios (35\% figura 9), cuyos valores superaron el $25 \%$ de vulnerabilidad. Los recursos comunales que están bajo dicho nivel de aceptabilidad son la infraestructura vial (caminos vecinales) y los puentes, así como las personas y todas aquellas viviendas que están emplazadas en áreas cercanas a las fuentes contaminantes.

En un segundo orden de prioridad, se encuentran 7 escenarios (41\%), que se clasificaron como inaceptables, pues se localizan según su valor de vulnerabilidad desde el $5,1 \%$ hasta el $25 \%$. Tales escenarios están compuestos por amenazas contaminantes al aire (polvo y malos olores), suelo (botaderos clandestinos), así como contaminación sónica generada por la actividad diaria de las fábricas cercanas a la comunidad.

Por consiguiente, se requiere desarrollar acciones prioritarias a corto plazo para su gestión, debido al alto impacto que estas tendrían sobre los recursos (infraestructura y personas) seleccionados.

Los recursos que se encuentran bajo este nivel de vulnerabilidad son las personas que habitan la comunidad, secciones de la red de caminos vecinales, puentes, la iglesia, el salón comunal, la escuela y las viviendas. En tercer orden de prioridad se localizan 4 escenarios que representan el 24\% del total (Figura 9), cuyo porcentaje de vulnerabilidad va desde el $0,3 \%$ hasta el $3 \%$, que los clasifica como "aceptables". Estos escenarios se relacionan con actos de delincuencia y falta de vigilancia sobre las personas y la infraestructura.

Según la definición de los criterios de aceptabilidad propuesta por Toro (2002), se establece que un escenario ubicado bajo dichos valores no implica una gravedad significativa; por lo tanto, se considera que no amerita la inversión de recursos y que no requiere acciones adicionales para la gestión sobre el factor de vulnerabilidad. 
Sin embargo, se considera pertinente propiciar las condiciones para el diálogo entre el Ministerio de Seguridad Pública, las empresas privadas ubicadas en la comunidad y los miembros de la comunidad para, así, definir estrategias de vigilancia y monitoreo en las zonas identificadas como inseguras por falta de vigilancia.

En la comunidad de Tablón de El Guarco se definieron un total de 14 escenarios de riesgo, relacionados con amenazas socionaturales (Figura 10). Estos, por su nivel de aceptabilidad, se clasificaron de la siguiente manera: un $20 \%$ como inadmisibles, un $45 \%$ como inaceptables, un $20 \%$ como tolerables y el restante $15 \%$ como aceptables.

En cuanto a las prioridades de intervención dada la gravedad relativa del riesgo que presentó cada escenario seleccionado en Tablón, se establece que en primer orden se definieron 3 escenarios (20\%), cuyos valores superan el $25 \%$ de vulnerabilidad, clasificándoles como inadmisibles. Dichos escenarios son de prioridad máxima de intervención, con recomendaciones para disminuir sus valores de vulnerabilidad.

En un segundo orden de prioridad, se encuentran 6 escenarios (45\%) que se clasificaron como inaceptables, pues su valor de vulnerabilidad va desde el 5,1\% hasta el $25 \%$ de vulnerabilidad.

Figura 10: Clasificación de aceptabilidad escenarios de riesgo Tablón

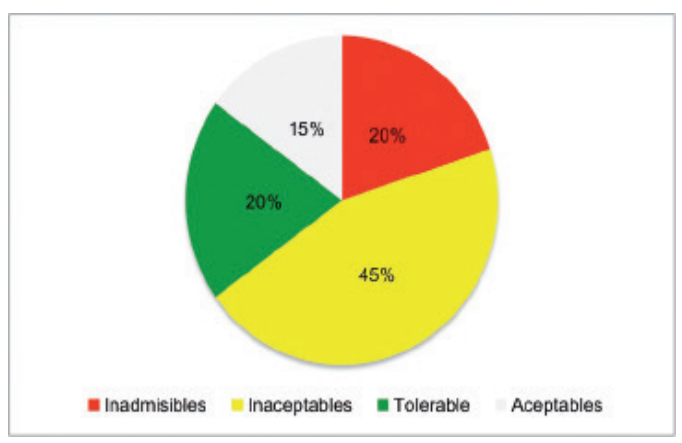

Fuente: Elaboración propia, 2013.

Tales escenarios están relacionados con amenazas como deslizamientos, desbordamientos de ríos y quebradas, además de erosión hídrica en las zonas de 
cultivo en terrenos con pendientes pronunciadas y fuertes. Con base en lo anterior, se propone desarrollar acciones de intervención prioritarias a corto plazo, debido al alto impacto que tales eventos tendrían sobre los recursos seleccionados (infraestructura, cultivos y personas).

En el tercer lugar de prioridad, se ubican 3 escenarios de riesgo (20\%) que se clasificaron como tolerables, pues su vulnerabilidad va desde el $3,1 \%$ hasta el $5 \%$. Ello implica que, aunque deben desarrollarse actividades para la gestión sobre el riesgo, tienen una prioridad de segundo nivel en comparación con los escenarios anteriores, lo cual permite diseñar estrategias de intervención a mediano plazo.

En cuarto lugar de prioridad se ubican 2 escenarios de riesgo que representan el $15 \%$ del total (Figura 10) y que se clasificaron como aceptables, dado que su condición de vulnerabilidad va desde el $0,3 \%$ hasta el $5 \%$.

Por consiguiente, la combinación de la frecuencia y las consecuencias no implican una gravedad significativa, por lo que no amerita la inversión de recursos y no requiere acciones adicionales para la gestión sobre el factor de vulnerabilidad considerado. Sin embargo, es necesario aclarar que tanto los escenarios clasificados como inadmisibles e inaceptables, así como los tolerables y aceptables, deben quedar sujetos a una constante vigilancia y monitoreo por parte de los pobladores de la comunidad por dos razones principales:

Primero, Aguilar \& Saborío (2006) proponen que la vulnerabilidad es inherente a todo ser vivo y está relacionada directamente con: las condiciones del ambiente físico y social que lo rodean y a factores de tiempo y espacio. Dado lo anterior, se establece que la vulnerabilidad estará matizada por todas las esferas que rodean la vida del ser humano y la forma en que éste se apodera del medio natural para su propio beneficio (Aguilar \& Saborio, 2006).

Por lo tanto, la vulnerabilidad no es constante en el tiempo y el espacio para una determinada amenaza, pues depende de las características y distancia del elemento vulnerable a la fuente de amenaza; ésta, en términos sociales, varía en función de edad, género, cultura o ingresos económicos (Peraldo, 2004).

Segundo, debido a que los porcentajes de vulnerabilidad se estimaron, en su mayoría, para escenarios de riesgo relacionados con amenazas socionaturales 
(deslizamientos y desbordamientos de ríos y quebradas) que están en estrecha relación con la manifestación de eventos hidrometeorológicos que impactan al área de estudio en período lluvioso, es necesario además definir estrategias de vigilancia, monitoreo y códigos de alerta temprana.

Lo anterior se debe principalmente a que tales eventos hidrometeorológicos (tormentas, lluvias torrenciales, huracanes en el mar Caribe) pueden variar en su intensidad y recurrencia año con año, dada la influencia del efecto directo del fenómeno de El Niño y La Niña para la región centroamericana, con lo cual también se podrían incrementar los eventos extremos (en la microcuenca del río Purires) con lluvias mayores a $1670 \mathrm{~mm}$ por año; es decir, 18\% por encima del promedio (Retana \& Villalobos, 2002).

\section{CONCLUSIONES}

En la comunidad de Coris de Cartago, se identificaron un total de 18 amenazas clasificadas por su origen en: antrópicas (56\%), socionaturales (39\%) y naturales $(5 \%)$.

También se determinó que la relación entre la cantidad y el tipo de amenazas antrópicas (especialmente aquellas asociadas a la presencia de sustancias peligrosas y contaminación) que afectan a los pobladores de Coris, está en estrecha relación con la cercanía geográfica de la comunidad respecto a la zona industrial de Cartago.

La clasificación de las amenazas por su nivel de significancia demostró que el $61 \%$ presentaron un alto nivel; el $33 \%$ un nivel medio y el restante $5 \%$, fueron amenazas de baja significancia dada su frecuencia, magnitud y área de impacto.

En Tablón de El Guarco, también se identificaron un total de 18 amenazas, clasificadas por su origen en: socionaturales (90\%) antrópicas (5\%) y naturales (55).

El número de amenazas socionaturales asociadas a deslizamientos y desbordamientos de ríos descrito en la comunidad de Tablón, está relacionado con el desarrollo histórico de actividades agropecuarias, las cuales han generado acelerados procesos de deforestación y erosión debido a cambios drásticos en el uso de la tierra, especialmente, en aquellas laderas con pendientes moderadas y fuertes de 
vocación forestal.

Asimismo, la clasificación de las amenazas por su nivel de significancia, evidenció que el $22 \%$ de éstas, se clasificaron con un alto nivel; el $67 \%$ como amenazas de nivel medio y el restante $11 \%$, de baja significancia.

Finalmente, se concluye que, si bien el análisis de vulnerabilidad se realizó puntualmente en dos comunidades rurales (con espacios geográficos muy bien diferenciados dadas sus actividades económicas, tipos de amenazas, usos de la tierra y características sociodemográficas), es importante aclarar e informar a los habitantes de Coris y Tablón, así como a otras comunidades vecinas, que éstas forman parte de una unidad geográfica definida por sus límites naturales como una microcuenca hidrográfica, cuyos componentes humanos, culturales y económicos, así como climáticos y ecológicos, agrícolas, edáficos, geológicos y geomorfológicos son propios e inigualables. Por lo tanto, toda modificación natural o antrópica, en sus partes altas, medias o bajas, generaría impactos y desequilibrios al resto de la microcuenca.

\section{BIBLIOGRAFÍA}

- Aguilar, M. \& Saborio, R. (2006): "La construcción histórica de la vulnerabilidad a inundaciones y deslizamientos en la Suiza, Turrialba". Univ. de Costa Rica. San José [Tesis Lic.].

- Álvarez, C., Calvo, M., Córdoba, J. \& Jirón, D. (2010): "Análisis de la erosión hídrica en los agroecosistemas café, helechos y pasto de la microcuenca alta del río Poás, Costa Rica". Univ. de Costa Rica. San José José [Tesis Lic.]

- Barquero, R. (2010): "Capítulo 3: Análisis de las intensidades y esbozo de la recurrencia de la sismicidad, en el área de Cartago", en: Peraldo, G. \& Acevedo, B. (ed.) 2010: Efemérides de la destrucción de la ciudad de Cartago. Cien años después (1910-2010). Ediciones Perro Azul, San José, págs. 49-58.Berrangé, J. \& Whittaker, J. (1977): "Reconnaissance geology of the Tapantí Quadrangle, Talamanca Cordillera, Costa Rica", en Institute of Geological Sciences, London [Informe interno].

- Brenes, A. \& González, P. (1998): “Gestión local, riesgo y vulnerabilidad en una 
localidad de la microcuenca 5 del río Virilla: Los Sitios, Moravia". Univ. de Costa Rica. San José [Tesis Lic.].

- Buch, M. \& Turcios, M. (2003): "Vulnerabilidad socioambiental: Aplicaciones para Guatemala". Universidad Rafael Landívar, Guatemala [Informe interno].

- Carrillo, S. \& Carrillo, M. (2005): "Estudio de la amenaza de inestabilidad de laderas y desarrollo de un escenario preliminar de la afectación de la infraestructura vital. Microcuenca del Río Purires, Provincia de Cartago, Costa Rica". Univ. de Costa Rica. San José [Tesis Lic.].

- CEPAL. (2005):"Elementos conceptuales para la prevención y reducción de daños originados por amenazas socionaturales. Cuatro experiencias en América Latina y el Caribe".LOM ediciones, Santiago de Chile.

- Céspedes, L. (2011): "Primer análisis de vulnerabilidad física de los principales centros poblados del área del proyecto MARAMEX-MANTARON, Región Junín". Escuela Académica Profesional de Ingeniería Geofísica. Universidad Mayor de San Marcos, Lima [Tesis Lic.].

- Córdoba, J. (2012): "Análisis de vulnerabilidad de las comunidades de Coris y Tablón en la microcuenca del río Purires, provincia de Cartago, Costa Rica". Univ. de Costa Rica. San José [Tesis M.Sc.].

- Denyer, P. \& Arias, O. (1991): "Estratigrafía de la región central de Costa Rica", en Rev. Geol. Amér. Central, N0.12, págs. 1 - 59.

- $\quad$ Escalante, J., Hilje, W., Leiva, D. \& Rivas, L. (2006): "Factores de vulnerabilidad social antedesastres en la comunidad de la cuenca del río Jucó, ubicada en el Valle de Orosí en la provincias de Cartago". Univ. de Costa Rica, San José [Tesis Lic.].

- Fernández, M. (1996): "Capítulo 1: Hacia un marco conceptual desde la perspectiva Latinoamericana”, en Fernández, M., [compiladora], 1996: Ciudades en riesgo: Degradación ambiental, riesgos urbanos y desastres. La Red, Lima, pág. 7-15.FICR. (2006): “¿Qué es el AVC? Introducción al análisis de vulnerabilidad y capacidad".Centro Regional de Referencia en Educación Comunitaria 
para la Prevención de Desastres, Federación Internacional de Sociedades de la Cruz Roja y de la Media Luna Roja. San José, 54 págs.

- Kholer, A., Jülich, S. \& Bloemertz, L. (2004): "Manual: El análisis de riesgo Una base para la gestión de desastres naturales".GTZ- Ministerio Federal de Cooperación Económica y Desarrollo. Eschborn, págs. 76.

- La Gaceta No 42 del 29 de febrero del 2000: Decreto Ejecutivo № 34728-S.

- La Gaceta Nº 8, 11 de enero de 2006: Ley Nacional de Emergencias y Prevención del Riesgo Nº 8488.

- Lavell, A. (1996): "Degradación ambiental, riesgo y desastre urbano. Problemas y conceptos: hacia la definición de una agenda de investigación", en: Fernández, M., [compiladora], 1996: Ciudades en riesgo: Degradación ambiental, riesgos urbanos y desastres. La Red, Lima. págs. 21-61.

- Lavell, A. (2004): "Sobre la gestión del riesgo: apuntes hacia una definición" La Red, Lima. págs. 23.

- Luengas, E. (2008): "Incorporación. La gestión del riesgo en instituciones educativas del municipio de los patios. Plan escolar para la gestión del riesgo". Dirección de prevención y atención de emergencias - DPAE. Bogotá, págs. 44.

- Melone, S. (2003): "Vulnerabilidad sísmica de edificaciones esenciales. Análisis de su construcción al riesgo sísmico". Escuela Técnica Superior. Barcelona [Tesis Ph.D.].

- Peraldo, G. (2004): "La novedad de lo constante: El proceso del desastre y su relación con la gestión territorial. Caso del distrito de Rivas, Pérez Zeledón, Costa Rica". Univ. de Costa Rica. San José [Tesis M.Sc.].

- Peraldo, G. \& Acevedo, B. (2010): "Efemérides de la destrucción de la ciudad de Cartago. Cien años después (1910-2010)”. Ediciones Perro Azul, San José.

- PRUGAM, (2010): "Mapa de uso de la tierra para la Gran Área Metropolitana.Escala 1: 25 000, Proyecto Regional Urbano de la Gran Área Metropolitana, 
San José".

- Fernández, M. (1996): "Capítulo 1: Hacia un marco conceptual desde la perspectivaRetana, J. \& Villalobos, R. (2002): "Eventos extremos meteorológicos en Linda Vista del Guarco, Cartago", en Tópicos Meteorológicos y Oceanográficos, N0. 9 (1), págs. $56-63$.

- Santos, M. (1986): "Por una geografía nueva". Espasa Calpe, Madrid.

- Sogreah Ingenierie, Gómez - Cajiao y Asociados \& Sinergia 69. (2000): "Plan de Manejo Integral de la Cuenca del Río Reventazón". Instituto Costarricense de Electricidad [Informe interno].

- Solano, J. \& Villalobos, R. (2001): "Aspectos fisiográficos aplicados a un bosquejo de regionalización geográfico climática de Costa Rica" en Tópicos Meteorológicos y Oceanográficos, N0. 8 (1), págs. 26 - 39.

- Toro, M. (2002): "Metodología para el análisis del riesgo y vulnerabilidad de sistemas de agua potable y saneamiento. Empresas públicas de Medellín E.S.P. Unidad estratégicas de negocios de aguas". III Curso internacional microzonificación y su aplicación en la mitigación de desastres. Lima.

- Trilla, J. (1992): "La educación no formal. Definición, conceptos básicos y ámbitos de aplicación". CEAC, Barcelona.

- Zúñiga, J. (1993): "Metodolgía para la determinación de la estabilidad de un río (caso del río Purires)" . Univ. de Costa Rica. San José [Tesis Lic.].

- Zusman, P. (2002): "Milton Santos. Su legado teórico y existencial (1926-2001)" en Documents d'Anàlisi Geogràfrica. N0.40, págs. 205-219. 